\title{
Update on the assessment of fluid responsiveness
}

\author{
Koichi Suehiro ${ }^{1}$ (1) \\ Received: 22 November 2019 / Accepted: 13 December 2019 / Published online: 7 January 2020 \\ (c) Japanese Society of Anesthesiologists 2020
}

Keywords Fluid responsiveness $\cdot$ Cardiac output $\cdot$ Stroke volume $\cdot$ Stroke volume variation $\cdot$ Pulse pressure variation

\section{Introduction}

Hemodynamic fluctuations, including hypotension and decreases in cardiac output (CO), frequently occur during general anesthesia and can lead to acute circulatory failure. Under these circumstances, fluid loading is the firstline therapeutic method. However, excessive fluid volume expansion can be harmful, especially in patients with heart disease. As patients with acute circulatory failure do not usually respond to fluid loading by increasing their $\mathrm{CO}$, numerous studies [1-3] have been conducted to develop tests that predict "fluid responsiveness". Although static indices, such as central venous pressure and pulmonary capillary wedge pressure, have been traditionally used for assessing patients' volume status, these indices have poor reliability for identifying fluid responders [4]. Dynamic indices, including pulse pressure variation (PPV) and stroke volume variation (SVV), have been recently used, and they provide better predictability for fluid responsiveness [5, 6]. Recent advances in minimally invasive hemodynamic monitoring allow for an increased clinical applicability of these indices [7-10]. Goaldirected therapy using dynamic indices decreases postoperative morbidity under various clinical conditions [11-13].

However, certain conditions, including arrhythmias and spontaneous breathing, impose some limitations for the clinical use of dynamic indices [14]. In particular, these indices have poor predictability for fluid responsiveness in patients with low tidal volumes $(<8 \mathrm{ml} / \mathrm{kg})$ or low driving airway pressures $\left(<20 \mathrm{~cm} \mathrm{H}_{2} \mathrm{O}\right)[15,16]$. Dynamic indices, including SVV and PPV, are calculated based on cyclic changes in intrathoracic pressure induced by positive

Koichi Suehiro

suehirokoichi@yahoo.co.jp

1 Department of Anesthesiology, Osaka City University Graduate School of Medicine, 1-5-7 Asahimachi, Abenoku, Osaka City, Osaka 545-8586, Japan pressure ventilation. When the change in intrathoracic pressure with low tidal volume ventilation is very small, the degree of respiratory variation of the preload in the right and left ventricles also becomes small. Thus, respiratory variations in stroke volume (SV) and pulse pressure are of limited use to assess volume status under this condition. This means that as lung-protective ventilation with low tidal volume $(6-7 \mathrm{ml} / \mathrm{kg})$ and positive end-expiratory pressure therapy $\left(5-10 \mathrm{cmH}_{2} \mathrm{O}\right)$ has become common during surgery, dynamic indices are less frequently used in the perioperative period [14]. Considering these weaknesses of dynamic indices, new interventions for assessing fluid responsiveness are required. Herein, the author briefly summarizes some currently developed interventions to predict fluid responsiveness.

\section{Recruitment maneuvers}

Recruitment maneuvers are common techniques to prevent or recover from lung collapse and to improve oxygenation in the intraoperative period. A recruitment maneuver induces an increase in intrathoracic and transpulmonary pressures, leading to increased pulmonary vascular resistance and decreased venous return, which results in a decrease in SV [17]. Hemodynamic changes induced by a recruitment maneuver depend on the patient's volume condition. A previous experimental study [18] showed that hemodynamic collapse caused by a recruitment maneuver was significantly higher under hypovolemic conditions than under hyper- and normovolemic conditions. A recent study by Biais et al. [17] investigated the reliability of SV changes induced by a recruitment maneuver for assessing fluid responsiveness. The study revealed that a decrease of $30 \%$ in SV during recruitment maneuver could predict an increase in SV after fluid loading (sensitivity: $88 \%$, specificity: 92\%). Recruitment maneuvers are also useful for assessing volume status 
under one-lung ventilation [19]. Specifically, a decrease in mean arterial pressure induced by a recruitment maneuver can predict fluid responsiveness in patients undergoing onelung ventilation (threshold: $9.5 \mathrm{mmHg}$, area under receiver operating characteristics [ROC] curve: 0.852). Observing hemodynamic changes during recruitment maneuver is likely to be a promising way to assess volume responsiveness. However, recruitment maneuvers should be performed cautiously for patients with a low $\mathrm{CO}$, because they increase the afterload in the right ventricle, which can be especially deleterious in such patients.

\section{End-expiratory occlusion test}

The end-expiratory occlusion test (EEOT) is a technique that comprises interrupting mechanical ventilation at the end of the expiration phase for 15-30 s and measuring subsequent hemodynamic changes [20]. Mechanical ventilation induces an increase in intrathoracic pressure and a decrease in venous return. When mechanical ventilation is interrupted at the expiration phase, the decrease in venous return is also interrupted, which leads to an increase in the preload of the ventricles. Therefore, an increase in SV induced by EEOT may indicate fluid responsiveness in mechanically ventilated patients.

A recent study [21] showed that EEOT can predict fluid responsiveness even in patients receiving lung-protective ventilation during surgery. In this study, an increase of 5\% in SV induced by EEOT identified fluid responders with a high sensitivity and specificity (100\% and $81 \%$, respectively). The ROC analysis revealed that the area under the curve (AUC) of SV increase by EEOT was significantly higher than that of PPV (0.91 versus $0.75, p<0.05)$. A recent meta-analysis indicated that the pooled area under the ROC curve for EEOT was 0.96 , with a pooled sensitivity and specificity of $86 \%$ and $91 \%$, respectively [22]. Although growing evidence has shown that EEOT can reliably detect patients with volume responsiveness, this technique has some limitations. First, EEOT requires a precise technique to measure SV and CO. Second, EEOT cannot be used in non-intubated patients nor can it be used in patients who cannot perform a respiratory hold for $15 \mathrm{~s}$. These limitations should be considered when EEOT is considered for use in clinical practice.

\section{Mini-fluid challenge}

The most effective method to test whether a patient is responsive to changes in preload is to assess the response of $\mathrm{SV}$ or CO to a fluid challenge. However, the traditional fluid bolus of "500 ml" is irreversible and can lead to a volume overload when performed repeatedly. This limitation has led to the recent development of a "mini"-fluid challenge of $100 \mathrm{ml}$ to assess fluid responsiveness.

A recent meta-analysis showed that the pooled area under the ROC curve for the mini-fluid challenge to predict fluid responsiveness was 0.91 , with a pooled sensitivity and specificity of $82 \%$ and $83 \%$, respectively [22]. Biais et al. [23] showed that SV changes induced by a mini-fluid bolus of $100 \mathrm{ml}$ (in $2 \mathrm{~min}$ ) were good predictors of fluid responsiveness (threshold: 6\%, sensitivity: $93 \%$, specificity: $85 \%$ ). Their ROC analysis revealed that the AUC of SV changes subsequent to a volume administration of $100 \mathrm{ml}(0.95,95 \%$ confidence interval: $0.90-0.99)$ was significantly greater than that induced by a $50 \mathrm{ml}$ fluid bolus $(0.83,95 \%$ confidence interval: $0.75-0.92)(p=0.01)$. This indicates that a fluid bolus of $50 \mathrm{ml}$ may be too small to generate significant changes enough to assess fluid responsiveness. Additionally, not only should the absolute volume of the fluid bolus be considered, but also its duration of administration [24]. Overall, there have been several ways in which fluid bolus administration has varied, including the amount of fluid in the bolus, duration of administration, and fluid type, which should be standardized in the future.

\section{Tidal volume challenge}

As mentioned above, dynamic indices have poor predictability in patients with a low tidal volume ventilation. Considering this weakness, "tidal volume challenge" can be a useful test to improve the ability of dynamic indices to identify fluid responders among such patients. This intervention involves transiently (in $1 \mathrm{~min}$ ) increasing the tidal volume from 6 to $8 \mathrm{ml} / \mathrm{kg}$ and assessing the changes in dynamic indices. Myatra et al. [25] showed that the absolute changes in PPV and SVV induced by a tidal volume challenge were good predictors of fluid responsiveness, with AUCs of 0.99 and 0.97 , respectively (threshold, PPV: $3.5 \%$, SVV: $2.5 \%$ ). In another recent study [26], the absolute changes in PPV and SVV after a tidal volume challenge showed acceptable predictabilities for fluid responsiveness, with high sensitivity and specificity even with patients in the prone position. Although the tidal volume challenge cannot be used in patients with spontaneous breathing, open-chest conditioning, or arrhythmia, this intervention can be a useful method to assess fluid responsiveness in the perioperative period under other circumstances.

\section{Future perspectives}

Considering the risk of volume overload, fluid administration should be carefully titrated according to patients' needs. As reported in this paper, several interventions exist for 
predicting fluid responsiveness. However, each of these techniques has its own limitations for clinical use. The choice of the technique depends on the capabilities available for hemodynamic monitoring and on the condition of the patient [27]. It is of great importance that the decision to administer fluid should be based on the presence of clinical signs of tissue hypoperfusion including hemodynamic instability and peripheral hypoperfusion. Volume expansion should not be performed solely based on the presence of fluid responsiveness [27]. These new interventions for predicting fluid responsiveness can be complementary, and clinicians should combine them according to patients' conditions. Future studies are needed to investigate the impact of the hemodynamic protocols including new hemodynamic interventions on the patients' outcomes.

\section{Compliance with ethical standards}

Conflict of interest K.S. has received speaker fees from Edwards Lifesciences and Otsuka Pharmaceutical Factory.

\section{References}

1. Okamura K, Nomura T, Mizuno Y, Miyashita T, Goto T. Preanesthetic ultrasonographic assessment of the internal jugular vein for prediction of hypotension during the induction of general anesthesia. J Anesth. 2019;33:612-9.

2. Mizunoya K, Saito H, Morimoto Y. Evaluation of external reference levels for central venous pressure measurements of severely obese patients in the supine position. J Anesth. 2018;32:558-64.

3. Hur M, Yoo S, Choi J-Y, Park S-K, Jung DE, Kim WH, Kim J-T, Bahk J-H. Positive end-expiratory pressure-induced increase in external jugular venous pressure does not predict fluid responsiveness in laparoscopic prostatectomy. J Anesth. 2018;32:316-25.

4. Guerin L, Monnet X, Teboul JL. Monitoring volume and fluid responsiveness: from static to dynamic indicators. Best Pract Res Clin Anaesthesiol. 2013;27:177-85.

5. Suehiro K, Rinka H, Ishikawa J, Fuke A, Arimoto H, Miyaichi T. Stroke volume variation as a predictor of fluid responsiveness in patients undergoing airway pressure release ventilation. Anaesth Intensive Care. 2012;40:767-72.

6. Cannesson M, Musard H, Desebbe O, Boucau C, Simon R, Henaine R, Lehot JJ. The ability of stroke volume variations obtained with Vigileo/FloTrac system to monitor fluid responsiveness in mechanically ventilated patients. Anesth Analg. 2009;108:513-7.

7. Sumiyoshi M, Maeda T, Miyazaki E, Hotta N, Sato H, Hamaguchi E, Kanazawa H, Ohnishi Y, Kamei M. Accuracy of the ClearSight ${ }^{\mathrm{TM}}$ system in patients undergoing abdominal aortic aneurysm surgery. J Anesth. 2019;33:364-71.

8. Maeda T, Hattori K, Sumiyoshi M, Kanazawa H, Ohnishi Y. Accuracy and trending ability of the fourth-generation FloTrac/ Vigileo system ${ }^{\mathrm{TM}}$ in patients undergoing abdominal aortic aneurysm surgery. J Anesth. 2018;32:387-93.

9. Juri T, Suehiro K, Kimura A, Mukai A, Tanaka K, Yamada T, Mori T, Nishikawa K. Impact of non-invasive continuous blood pressure monitoring on maternal hypotension during cesarean delivery: a randomized-controlled study. J Anesth. 2018;32:822-30.

10. Takeda C, Takeuchi M, Mizota T, Yonekura H, Nahara I, Kuwauchi A, Joo WJ, Kawasaki Y, Kawakami K. Utilization of arterial pulse waveform analysis during non-cardiac surgery in Japan: a retrospective observational study using a nationwide claims database. J Anesth. 2019;33:159-62.

11. Som A, Maitra S, Bhattacharjee S, Baidya DK. Goal directed fluid therapy decreases postoperative morbidity but not mortality in major non-cardiac surgery: a meta-analysis and trial sequential analysis of randomized controlled trials. J Anesth. 2017;31:66-81.

12. Mizunoya K, Fujii T, Yamamoto M, Tanaka N, Morimoto Y. Twostage goal-directed therapy protocol for non-donor open hepatectomy: an interventional before-after study. J Anesth. 2019 (in press).

13. Coeckelenbergh S, Zaouter C, Alexander B, Cannesson M, Rinehart J, Duranteau J, Van der Linden P, Joosten A. Automated systems for perioperative goal-directed hemodynamic therapy. J Anesth. 2019 (in press).

14. Mair S, Tschirdewahn J, Gotz S, Frank J, Phillip V, Henschel B, Schultheiss C, Mayr U, Noe S, Treiber M, Schmid RM, Saugel B, Huber W. Applicability of stroke volume variation in patients of a general intensive care unit: a longitudinal observational study. J Clin Monit Comput. 2017;31:1177-87.

15. Suehiro K, Okutani R. Influence of tidal volume for stroke volume variation to predict fluid responsiveness in patients undergoing one-lung ventilation. J Anesth. 2011;25:777-80.

16. Muller L, Louart G, Bousquet PJ, Candela D, Zoric L, de La Coussaye JE, Jaber S, Lefrant JY. The influence of the airway driving pressure on pulsed pressure variation as a predictor of fluid responsiveness. Intensive Care Med. 2010;36:496-503.

17. Biais M, Lanchon R, Sesay M, Le Gall L, Pereira B, Futier E, Nouette-Gaulain K. Changes in stroke volume induced by lung recruitment maneuver predict fluid responsiveness in mechanically ventilated patients in the operating room. Anesthesiology. 2017;126:260-7.

18. Nielsen J, Nilsson M, Freden F, Hultman J, Alstrom U, Kjaergaard J, Hedenstierna G, Larsson A. Central hemodynamics during lung recruitment maneuvers at hypovolemia, normovolemia and hypervolemia. A study by echocardiography and continuous pulmonary artery flow measurements in lung-injured pigs. Intensive Care Med. 2006;32:585-94.

19. Kang WS, Oh CS, Park C, Shin BM, Yoon TG, Rhee KY, Woo NS, Kim SH. Diagnosis accuracy of mean arterial pressure variation during a lung recruitment maneuver to predict fluid responsiveness in thoracic surgery with one-lung ventilation. Biomed Res Int. 2016;2016:3623710.

20. Gavelli F, Teboul JL, Monnet X. The end-expiratory occlusion test: please, let me hold your breath! Crit Care. 2019;23:274.

21. Biais M, Larghi M, Henriot J, de Courson H, Sesay M, NouetteGaulain K. End-expiratory occlusion test predicts fluid responsiveness in patients with protective ventilation in the operating room. Anesth Analg. 2017;125:1889-955.

22. Messina A, Dell'Anna A, Baggiani M, Torrini F, Maresca GM, Bennett V, Saderi L, Sotgiu G, Antonelli M, Cecconi M. Functional hemodynamic tests: a systematic review and a metanalysis on the reliability of the end-expiratory occlusion test and of the mini-fluid challenge in predicting fluid responsiveness. Crit Care. 2019;23:264.

23. Biais M, de Courson H, Lanchon R, Pereira B, Bardonneau G, Griton M, Sesay M, Nouette-Gaulain K. Mini-fluid challenge of $100 \mathrm{ml}$ of crystalloid predicts fluid responsiveness in the operating room. Anesthesiology. 2017;127:450-6.

24. Toscani L, Aya HD, Antonakaki D, Bastoni D, Watson X, Arulkumaran N, Rhodes A, Cecconi M. What is the impact of the 
fluid challenge technique on diagnosis of fluid responsiveness? A systematic review and meta-analysis. Crit Care. 2017;21:207.

25. Myatra SN, Prabu NR, Divatia JV, Monnet X, Kulkarni AP, Teboul JL. The changes in pulse pressure variation or stroke volume variation after a "tidal volume challenge" reliably predict fluid responsiveness during low tidal volume ventilation. Crit Care Med. 2017;45:415-21.

26. Messina A, Montagnini C, Cammarota G, Giuliani F, Muratore L, Baggiani M, Bennett V, Della Corte F, Navalesi P, Cecconi M. Assessment of fluid responsiveness in prone neurosurgical patients undergoing protective ventilation: role of dynamic indices, tidal volume challenge, and end-expiratory occlusion test. Anesth Analg. 2019 (in press).

27. Monnet X, Marik PE, Teboul JL. Prediction of fluid responsiveness: an update. Ann Intensive Care. 2016;6:111.

Publisher's Note Springer Nature remains neutral with regard to jurisdictional claims in published maps and institutional affiliations. 